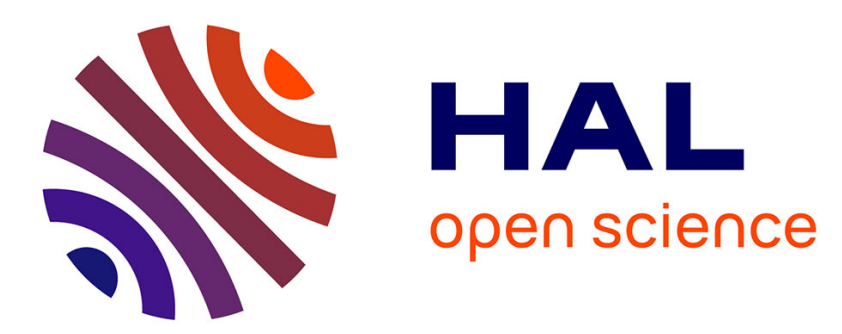

\title{
Nonequilibrium radiation from a recombining nitrogen plasma
}

\author{
Augustin Tibère-Inglesse, Sean Mcguire, Christophe O Laux
}

\section{To cite this version:}

Augustin Tibère-Inglesse, Sean Mcguire, Christophe O Laux. Nonequilibrium radiation from a recombining nitrogen plasma. 2018 AIAA Aerospace Sciences Meeting, Jan 2018, Kissimmee, United States. 10.2514/6.2018-0241 . hal-01866394

\section{HAL Id: hal-01866394 https://hal.science/hal-01866394}

Submitted on 28 Feb 2020

HAL is a multi-disciplinary open access archive for the deposit and dissemination of scientific research documents, whether they are published or not. The documents may come from teaching and research institutions in France or abroad, or from public or private research centers.
L'archive ouverte pluridisciplinaire HAL, est destinée au dépôt et à la diffusion de documents scientifiques de niveau recherche, publiés ou non, émanant des établissements d'enseignement et de recherche français ou étrangers, des laboratoires publics ou privés. 


\title{
Nonequilibrium radiation from a recombining nitrogen plasma
}

\author{
$\underline{\text { Augustin Tibère-Inglesse }}{ }^{1,2}$, Sean McGuire ${ }^{2}$ \& Christophe O. Laux ${ }^{2}$ \\ ${ }^{1}$ Ariane Group, 66 Route de Verneuil, 78130 Les Mureaux \\ ${ }^{2}$ Laboratoire EM2C, CNRS UPR288, CentraleSupélec, Université Paris-Saclay, 10 rue Joliot-Curie, \\ 91190, Gif-sur-Yvette, France
}

Previous experiments performed at Stanford University studied the properties of a recombining nonequilibrium nitrogen plasma at atmospheric pressure. They used an inductively coupled plasma torch to create an equilibrium plasma and then imposed rapid cooling within a water-cooled tube in order to force rapid recombination and generate non-equilibrium distributions of ground and excited species. Emission spectroscopy was used to measure the plasma properties. Comparisons with numerical simulations showed that CFD codes did not correctly predict the temperature drop and the radiative flux leaving the plasma. This paper discusses a re-analysis of these experiments using new temperature measurements performed using Raman spectroscopy. A key result is that the measured temperature drop due to the imposed cooling is even higher than that measured by prior emission spectroscopy measurements. In addition to the Raman measurements, optical emission measurements were also performed and used to measure the atomic nitrogen density. From these measurements, we show that a large portion of the plasma enthalpy is stored in non-recombined nitrogen atoms. This is an important consideration for CFD codes looking to model atmospheric reentry dynamics. 


\section{Introduction}

During atmospheric entry, a capsule enters the atmosphere of a planet (Earth, Mars...) at a velocity that can exceed 10 $\mathrm{km} / \mathrm{s}$. The strong shockwave that forms in front of the capsule heats the gas to temperatures that can exceed 10,000K. The high temperature of the gas leads to ionization, molecular dissociation, excitation and intense gas radiation. The study of the radiative and convective heat transfer of such a plasma is critical for the design of the heat shield (or thermal protection system, TPS) of a reentry capsule. These heatshields are carbon-based and represent a significant constraint for the mission, particularly in regards to their cost and weight. The heat flux to the forebody of the capsule has been the primary focus of past research and is now relatively well understood [1] [2]. However, predictions of the heat flux to the afterbody surface suffer from large uncertainties. This is due mainly to two factors. The first is the presence of significant quantities of carbon in the plasma, introduced via the ablative heat shield in the front of the capsule. The carbon sublimates from the forebody heat shield on the capsule and recombines in the flow, forming molecules such as $\mathrm{CN}$ and $\mathrm{CO}$, that radiate in the afterbody region. This is currently the focus of some studies; see for example [3] [4] [5]. The second phenomenon is the expansion of the plasma into the afterbody region. This expansion rapidly cools the plasma, forces plasma recombination and departure from chemical equilibrium. The prediction of this nonequilibrium and the resulting radiation from such a plasma is not accurately modeled at the moment. Experiments conducted at Stanford University between 1995 and 2003 in atmospheric pressure recombining nitrogen plasmas showed that the radiation of certain transitions could emit up to 30 times more than the corresponding equilibrium radiation [6] [7]. CFD simulations were also performed to reproduce the $\mathrm{N}$ recombination and gas cooling but were not in agreement with the measurements [8] [9] as shown in Figure 1. In particular, the CFD calculations predicted that the gas temperature decreases by about $400 \mathrm{~K}$ between the tube entrance and exit for a tube length of $15 \mathrm{~cm}$. In contrast, the measurements show more than $2400 \mathrm{~K}$ of temperature decrease.
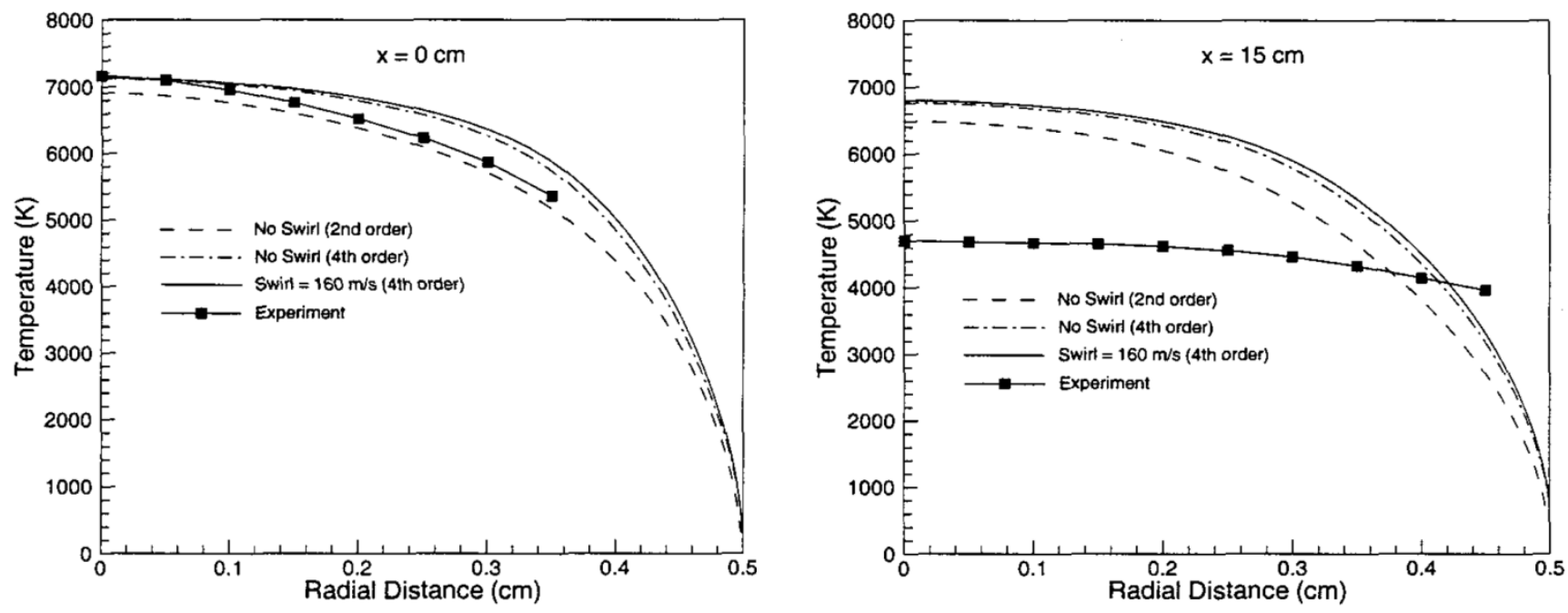

Figure 1: CFD simulations vs experimental results for several CFD simulations. Figures taken from

The combined effect of these two phenomena leads to large uncertainties on the heat flux to the afterbody surface. For earth reentry, simulations performed by Johnston et al [2] [10] show that uncertainties on the radiation heat flux to the afterbody can be up to $100 \%$ for velocities around $14 \mathrm{~km} / \mathrm{s}$. The velocity in the case of a real Mars reentry scenario could be higher, particularly for a Mars aerocapture mission [11]. This would increase the uncertainty resulting from these phenomena.

Although the heat flux per unit area to the afterbody surface is lower than on the forebody (for example, for the Stardust reentry, the total heat flux on the afterbody is about $20 \mathrm{~W} / \mathrm{cm}^{2}$ as compared to $100 \mathrm{~W} / \mathrm{cm}^{2}$ in the forebody), the afterbody surface represents approximately $2 / 3$ of the total area, and therefore significant heating may occur on the afterbody. Uncertainties on these heat fluxes cause an overestimation of the TPS shield due to design margins, which can lead to a significant cost increase and added weight. This, in turn, can affect the feasibility of a mission. A fundamental study of the mechanisms governing the plasma dynamics in the afterbody of a spacecraft is therefore needed in order to 
reduce these uncertainties. This work will focus on the radiation of a non-equilibrium recombining plasma flow to better understand this mechanism.

In this paper, we revisit the previous experiments conducted by Laux, Gessman et al [6] [7]. To this end, we have now implemented a new Raman scattering diagnostic to complement emission spectroscopy measurements. We used our $50 \mathrm{~kW}$ plasma torch to supply a high enthalpy plasma, at conditions reproducing those of the Stanford experiments. The plasma is then cooled in a water-cooled tube to force the plasma to recombine, resulting in a nonequilibrium plasma at the exit of the tube. We use emission and Raman spectroscopy to study the departure from equilibrium and the resulting radiation from the plasma. This experiment provides a simple test case for a recombining plasma and can be used to validate CFD codes: in particular, it serves as a validation case for the chemical kinetics being used in the CFD codes. We performed experiments for a variety of conditions, ranging from equilibrium to nonequilibrium cases, in order to try and assess the impact of the chemical kinetics on the plasma properties.

\section{Experimental setup}

The plasma torch facility is a TAFA Model 66 inductively coupled plasma (ICP) torch powered by a $120 \mathrm{kVA}$ radio frequency LEPEL Model T-50-3 power supply. The power supply operates at $4 \mathrm{MHz}$ and can supply a maximum of $12 \mathrm{kV}$ DC and 7.5 A to the oscillator plates. Details of the plasma torch facility are provided in previous publications [4] [7] [12]. The plasma produced within the torch exits through a 1-cm diameter nozzle at a speed of approximately $600 \mathrm{~m} / \mathrm{s}$. The plasma studied here are comprised of $90 \mathrm{slpm}$ of $\mathrm{N}_{2}$ or air premixed with $50 \mathrm{slpm}$ of argon to provide stable operating conditions for the torch. A water-cooled tube $(15 \mathrm{~cm}$ length) made of brass is mounted on top of the plasma torch. Instead of creating a strong expansion as with a reentering capsule, the plasma is forced to recombine by imposing rapid cooling via the cold wall (maintained at approximately $300 \mathrm{~K}$ ). This setup is therefore intended as a simplified experimental model of a real reentry scenario. Details of the experimental setup are presented in figure 2 . We will compare the results between the $\mathrm{N}_{2} / \mathrm{Ar}$ and the Air/Ar plasmas.

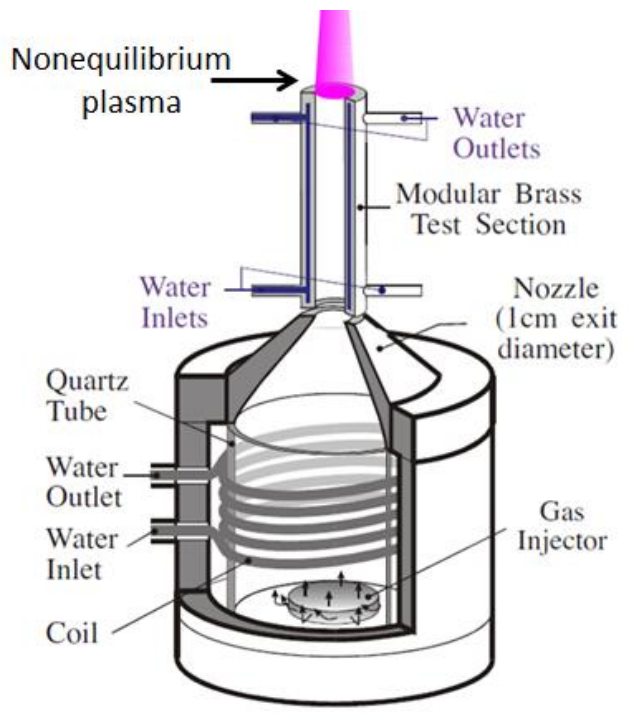

Figure 2: Water-cooled test-section mounted on the top of the plasma torch

Measurements were performed for several tube lengths:

- A 0 -cm tube length corresponds to measurements directly at the exit of the torch nozzle (no water-cooled tube). It has been shown that the plasma is in equilibrium at this location [7] [13]. The plasma temperature is measured by optical emission spectroscopy (see Figure 3) of atomic lines [12]. Measured molecular spectra are then compared with theoretical ones generated using the radiation code SPECAIR [14] assuming the equilibrium measured temperature and the known pressure $(1 \mathrm{~atm})$. The good agreement between theory and experiment validates the equilibrium assumption. Raman measurements recently performed in our group for these conditions have also confirmed the temperature obtained by emission spectroscopy [14].

- At 10-cm and 15-cm tube lengths, temperature measurements are performed using both emission and Raman spectroscopy. At $10 \mathrm{~cm}$, the temperature is measured from the absolute emission of atomic lines. The temperature at $15 \mathrm{~cm}$ is measured using emission spectroscopy of $\mathrm{N}_{2}{ }^{+}$rotational lines using the procedure 
detailed in [15]. Measured $\mathrm{N}_{2}$ first positive spectra are compared with theoretical ones computed using SPECAIR at the measured temperature. If the theoretical and measured spectra are not in agreement, it is possible to adjust the population of all emitting vibrational levels of the $\mathrm{N}_{2} \mathrm{~B}$ electronic state in SPECAIR for a given transition ( $\mathrm{N}_{2}$ first positive in our case). This is done using an overpopulation factor for the vibrational level defined as $\rho_{v}=\frac{\left.n_{v} \text { (measured }\right)}{\left.n_{v} \text { (equilibrium) }\right)}$ where $v$ is the vibrational level considered and $\mathrm{n}$ the population of this level.

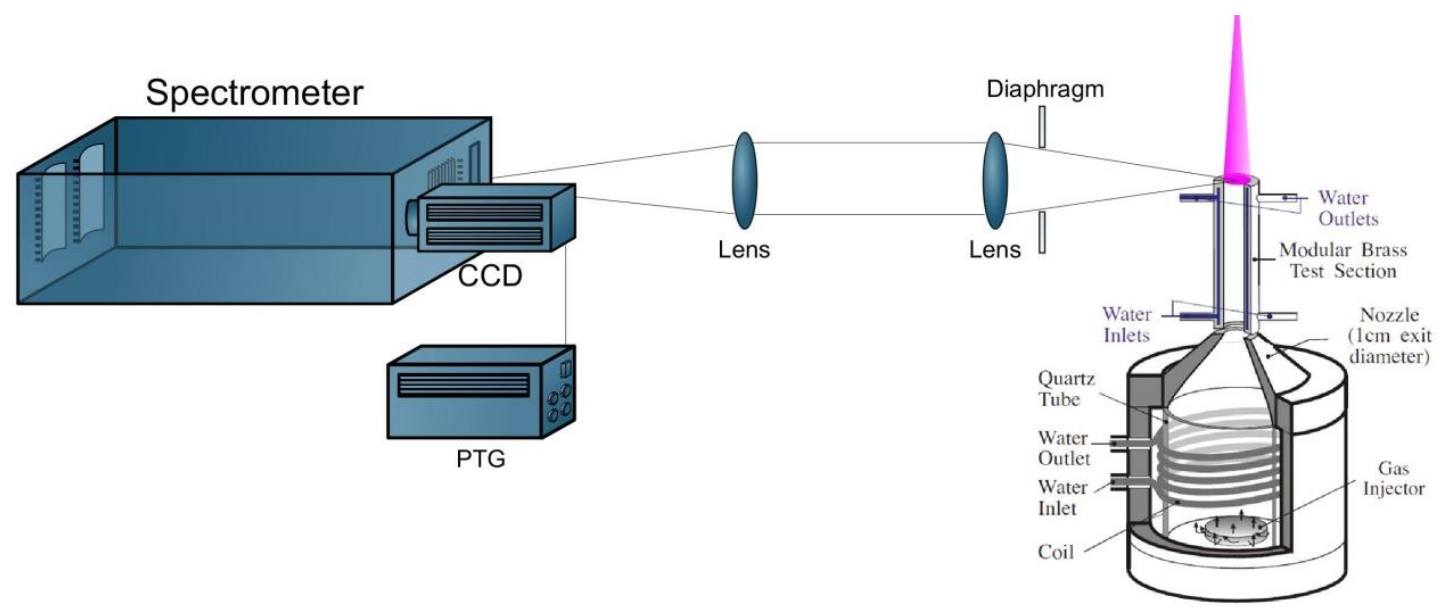

Figure 3: Experimental setup for emission spectroscopy

All spectra measured using emission spectroscopy are calibrated from pixel counts to absolute emission intensity with an Optronics Laboratories tungsten lamp (model OL550). All spectra are Abel-inverted to get the spatially resolved local plasma emission.

To provide an independent confirmation of the gas temperature inferred from emission spectroscopy, Raman scattering measurements have been performed to measure the ground state temperature of $\mathrm{N}_{2}$. The measurements performed by Laux, Gessman et al used emission spectroscopy and gave only temperatures of excited states of atoms (at 0 and 10 $\mathrm{cm}$ ) or ions (at $15 \mathrm{~cm}$ ) of the gas. Raman spectroscopy has allowed us to measure the temperature of the fundamental state of nitrogen: given that the bulk of the gas will be in this ground state, this measurement is more representative of the gas temperature. The experimental setup for Raman spectroscopy is presented in Figure 4 and the detailed measurement procedure is given in Ref [14]. In summary, we performed vibrational Raman spectroscopy in the UV region of the spectrum. A frequency-doubled Continuum dye laser was used to generate a $10 \mathrm{~ns}, 30 \mathrm{~mJ}$ ultraviolet pulse at $281 \mathrm{~nm}$. Rhodamine 590 dye was used to supply a laser beam at $562 \mathrm{~nm}$ that was then frequency doubled to produce the final output at $281 \mathrm{~nm}$. While the quadrupled output of a Nd:YAG laser at $266 \mathrm{~nm}$ may have sufficed for the measurements, the ability to tune the dye laser offered the ability to avoid potential laser-induced interferences in the UV. The wavelength used for these measurements, namely $281 \mathrm{~nm}$, represented a compromise between avoiding laser-induced interferences and maximizing laser power. This technique is sensitive to both the rotational and vibrational temperature of the $\mathrm{N}_{2}$ ground state. The results suggest that both temperatures are in equilibrium, so only one temperature will be given in the results section. Indeed, the model and the experiments are in better agreement when both temperatures are equal [14]. Moreover, rotational relaxation at atmospheric pressure is very fast so we assume that rotational and translational temperatures are equal. 


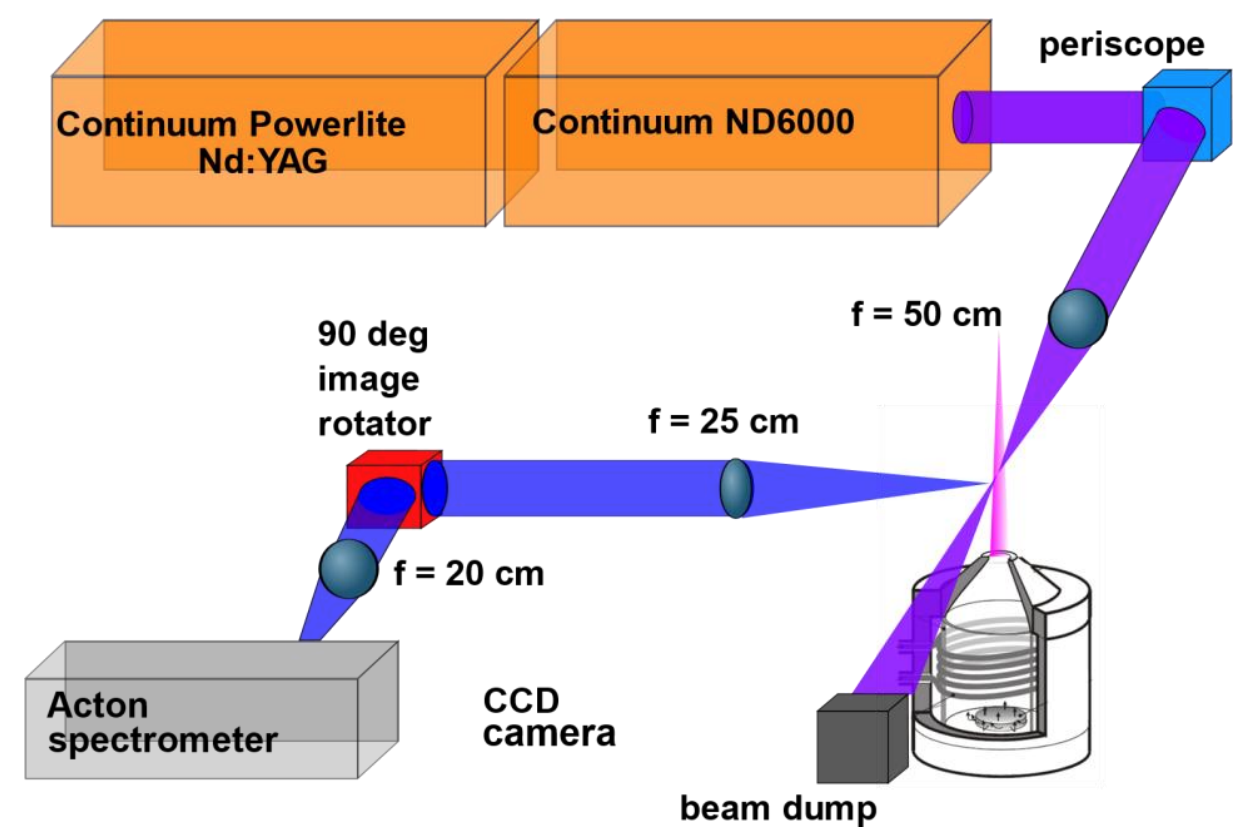

Figure 4: Experimental setup for Raman UV scattering measurements. The laser is focused above the torch exit. The periscope consists of two quartz prisms for adjusting the height and direction of the laser beam. The $\mathbf{9 0}^{\circ}$ image rotator serves to horizontally align the laser beam with the spectrometer entrance slit.

\section{- $0 \mathrm{~cm}$ (water-cooled tube inlet)}

\section{Results}

Temperature profiles in both nitrogen and air plasmas are presented in figure 5. The profile labeled "Gessman et al" corresponds to the temperature measured by Gessman et al [16] of a $\mathrm{N}_{2} / \mathrm{Ar}$ plasma using emission spectrocopy and for the same flow rate as used in this paper. Results from Raman spectroscopy are also presented in Fig. 5. The temperature measurements using emission spectroscopy are performed using the absolute intensity of the atomic nitrogen line at $746 \mathrm{~nm}$ (labeled N746) in the case of the nitrogen plasma, and O777 in the case of the air plasma. Also, temperature measurements using N742, N744, Ar751 and Ar763 were performed and confirmed these temperature measurements: these are not presented here for the sake of clarity. Results from Raman spectroscopy are also presented. At these high temperatures, the gas is highly dissociated, and the density is low, leading to a small population of $\mathrm{N}_{2}(\mathrm{X})$. Therefore, the Raman signal is weak, and the associated uncertainty is large.

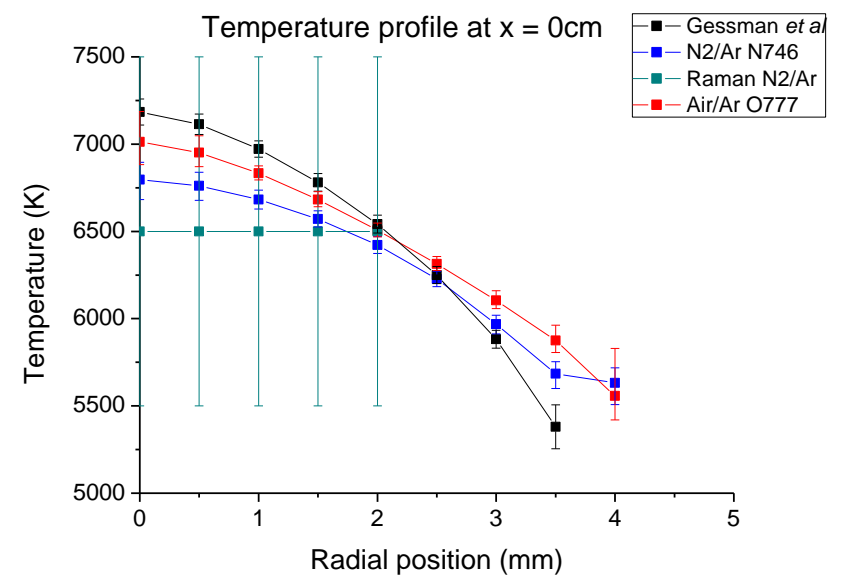

Figure 5: temperature profiles measured using both emission and Raman spectroscopy at tube inlet $(x=0 \mathrm{~cm})$. 
The temperature measurements of Gessman et al and our own for an $\mathrm{N}_{2} / \mathrm{Ar}$ plasma are in good agreement. A slight difference is observed between the two sets of results, although the torch power and the mass flow rates were identical in both experiments. This difference can be attributed to different injection conditions in the plasma torch: in the experiments of Gessman et al, $50 \%$ of the gas was injected in swirl mode, and 50\% in radial mode, whereas in the present experiments we used $20 \%$ swirl / $80 \%$ radial. Swirl can slightly change the temperature profiles, as seen from the computations shown in Fig. 1.

The Raman measurements for Air/Ar using Raman spectroscopy are not presented as they give the same profile as for the $\mathrm{N}_{2} / \mathrm{Ar}$ plasma. Temperature profiles of the Air/Ar plasma obtained using emission and Raman spectroscopy are in agreement to within the uncertainty bars. The good agreement between temperature measurements using different atomic transitions and Raman scattering gives confidence that the plasma is in equilibrium.

\section{- $10 \mathrm{~cm}$ water-cooled tube exit}

Temperature profiles at $10 \mathrm{~cm}$ were performed using the same techniques as those used at $0 \mathrm{~cm}$ and are presented in figure 6.

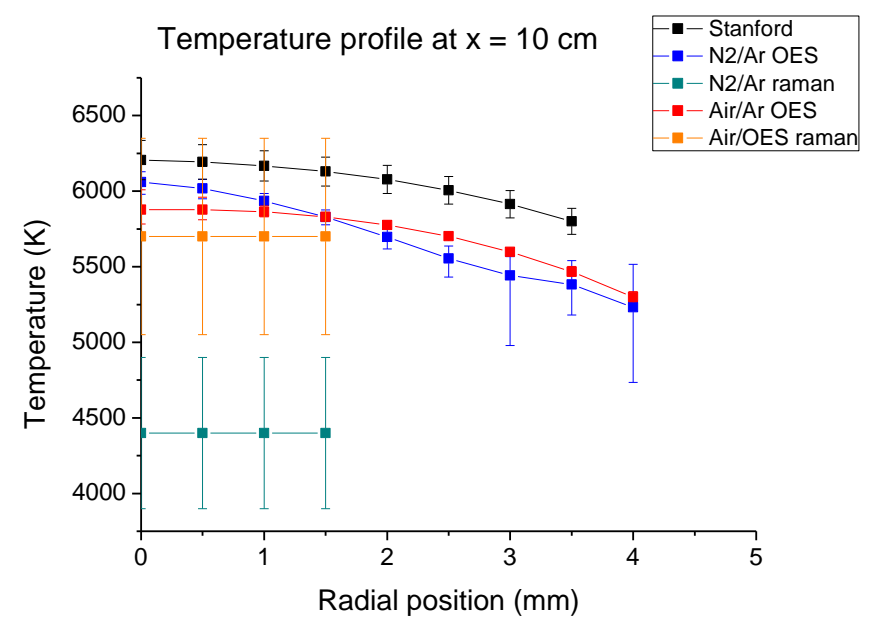
Figure 6: temperature profiles measured using both emission and Raman spectroscopy at $10 \mathrm{~cm}$ water-cooled tube exit.

The temperature profiles of Gessman et al and our emission measurements for the $\mathrm{N}_{2} / \mathrm{Ar}$ plasma are in good agreement again as expected. Both Raman and emission spectroscopy temperature profiles remain in good agreement for the Air/Ar plasma. However, the temperature profiles measured using emission and Raman spectroscopy now differ for the case of the $\mathrm{N}_{2} / \mathrm{Ar}$ plasma: this is the case despite the relatively large uncertainty bars. This was unanticipated. Note that the temperatures measured using Raman spectroscopy are more representative of the temperature of the gas because they are a measurement of the ground state of $\mathrm{N}_{2}$. The emission measurements are in agreement with those of Gessman et al. However, the Raman measurements show that the bulk gas temperature is much lower than previously thought. Also note that the Raman measurements, rather than reducing the discrepancy with the CFD model predictions in Fig. 1, amplify this discrepancy.

\section{- $15 \mathrm{~cm}$ water-cooled tube exit}

Temperature profiles at $15 \mathrm{~cm}$ are presented in figure 7. 


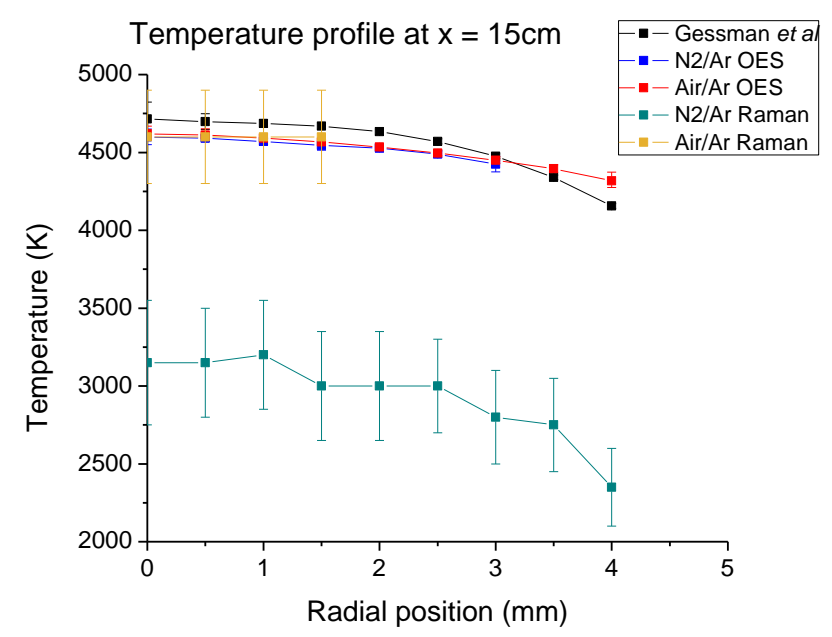

Figure 7: temperature profiles measured using both emission and Raman spectroscopy at $15 \mathrm{~cm}$ water-cooled tube exit.

Once again, all temperature profiles are in good agreement with the exception of the Raman measurement in the $\mathrm{N}_{2} / \mathrm{Ar}$ plasma. The differences between the temperatures measured using Raman spectroscopy and emission spectroscopy for the $\mathrm{N}_{2} / \mathrm{Ar}$ plasma are now even more pronounced than for the case at $10 \mathrm{~cm}$. Emission spectroscopy measurements are based on emission from the $\mathrm{N}_{2}{ }^{+} \mathrm{B}$ state. Previous work suggested that the ion may recombine before it equilibrates to the background rotational/translational temperature [17]. This would mean that this emission is not representative of the bulk gas temperature. Indeed, the Raman measurements show that the bulk gas temperature is much lower than previously thought. This confirms that a recombination mechanism in the $\mathrm{N}_{2} / \mathrm{Ar}$ plasma causes a rapid temperature decrease that is not accounted for in the CFD models (Fig. 1). Note that, for the case of the Air/Ar plasma, the temperatures measured using both techniques provide similar results and give confidence in the temperature measured by Raman for the $\mathrm{N}_{2} /$ Ar case.

In both the air and $\mathrm{N}_{2}$ plasma we measured $\mathrm{N}_{2}$ first positive emission in the visible wavelength range for comparison with SPECAIR modeling. Figure 8 shows the results. We computed with SPECAIR the theoretical spectrum for an air/Ar and a $\mathrm{N}_{2} /$ Ar plasma under local thermodynamic equilibrium conditions at the measured temperature and 1 atm. For the $\mathrm{N}_{2} /$ Ar plasma case, we computed two theoretical spectra: one with the temperature measured using emission spectroscopy, and the second with the temperature measured using Raman spectroscopy.

Theoretical and measured spectra at the exit of a $15 \mathrm{~cm}$ tube
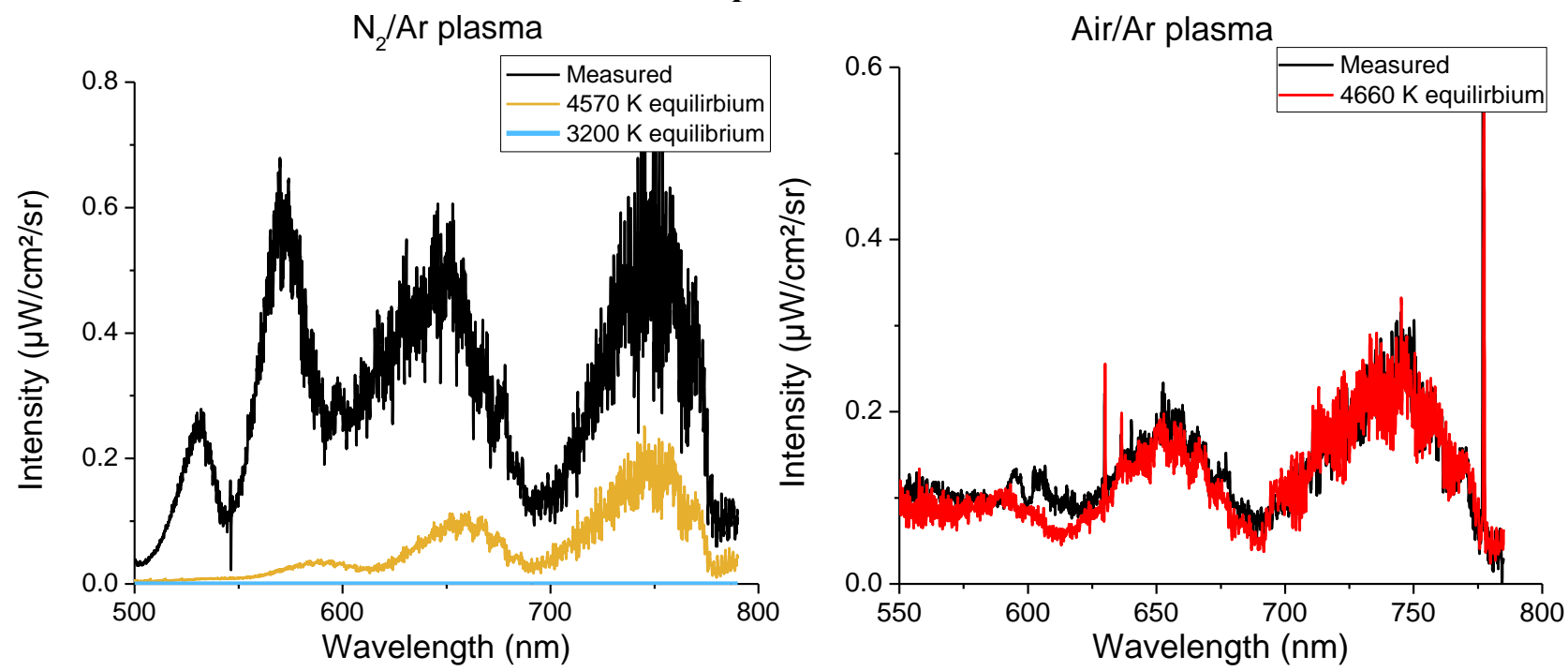

Figure 8: calibrated measured spectra for the $\mathrm{N}_{2} / \mathrm{Ar}$ and Air/Ar plasmas and the corresponding computed spectra using SPECAIR, assuming equilibrium at the exit of the $15 \mathrm{~cm}$ tube. The measured spectra are Abelinverted and only the emission of the $0.5 \mathrm{~mm}$ thick center slab is plotted. 
There is good agreement between the measured and computed spectra for the case of the Air/Ar plasma, confirming equilibrium at the exit of the $15 \mathrm{~cm}$ tube. However, there is a large discrepancy for the case of the $\mathrm{N}_{2} / \mathrm{Ar}$ plasma. Note that this discrepancy is large even with the higher temperature obtained using emission spectroscopy. This shows that the plasma is not in equilibrium. Considering only the $\mathrm{N}_{2}$ first positive transition, it appears that the measured radiation from this transition is about 5 times more than the equilibrium value if a temperature of $4570 \mathrm{~K}$ is assumed (temperature measured with emission). If we assume that the plasma is at $3200 \mathrm{~K}$, which corresponds to the temperature obtained using Raman spectroscopy, the measured radiation is now about 10000 times more than equilibrium. In term of CFD simulations, this shows that the plasma is not in equilibrium and that the temperature is not the only relevant physical quantity to be modeled. A vibrationally specific collisional-radiative model is essential to correctly predict the radiative heat flux. Note that Gessman et al showed, for the case of the Air/Ar plasma, that the additional presence of oxygen tends to keep the recombining plasma at equilibrium via the recombination reaction: $N+O+M \leftrightarrow N O+M$ [16].

To quantify the degree of departure from equilibrium in the vibrational levels of the $\mathrm{N}_{2} \mathrm{~B}$ state, we used SPECAIR and altered the population of the vibrational levels of $\mathrm{N}_{2} \mathrm{~B}$ to reproduce the experimental spectrum. This was done by using the previously defined overpopulation factor $\rho_{v}$ to increase the population of a given vibrational level. The experimental spectrum was reconstructed by introducing overpopulation factors for the various contributing vibrational levels. This was done using an optimization algorithm. The results of the measured population and the corresponding spectra are presented in figure 9. Note that we used the temperature measured using Raman as it is the most representative of the gas temperature. Note that the level $\mathrm{N}_{2}{ }^{+}\left(\mathrm{A}^{2} \Sigma_{\mathrm{g}}{ }^{+}\right)$is also overpopulated as the transition $\mathrm{N}_{2}{ }^{+}$Meinel is stronger than at equilibrium.

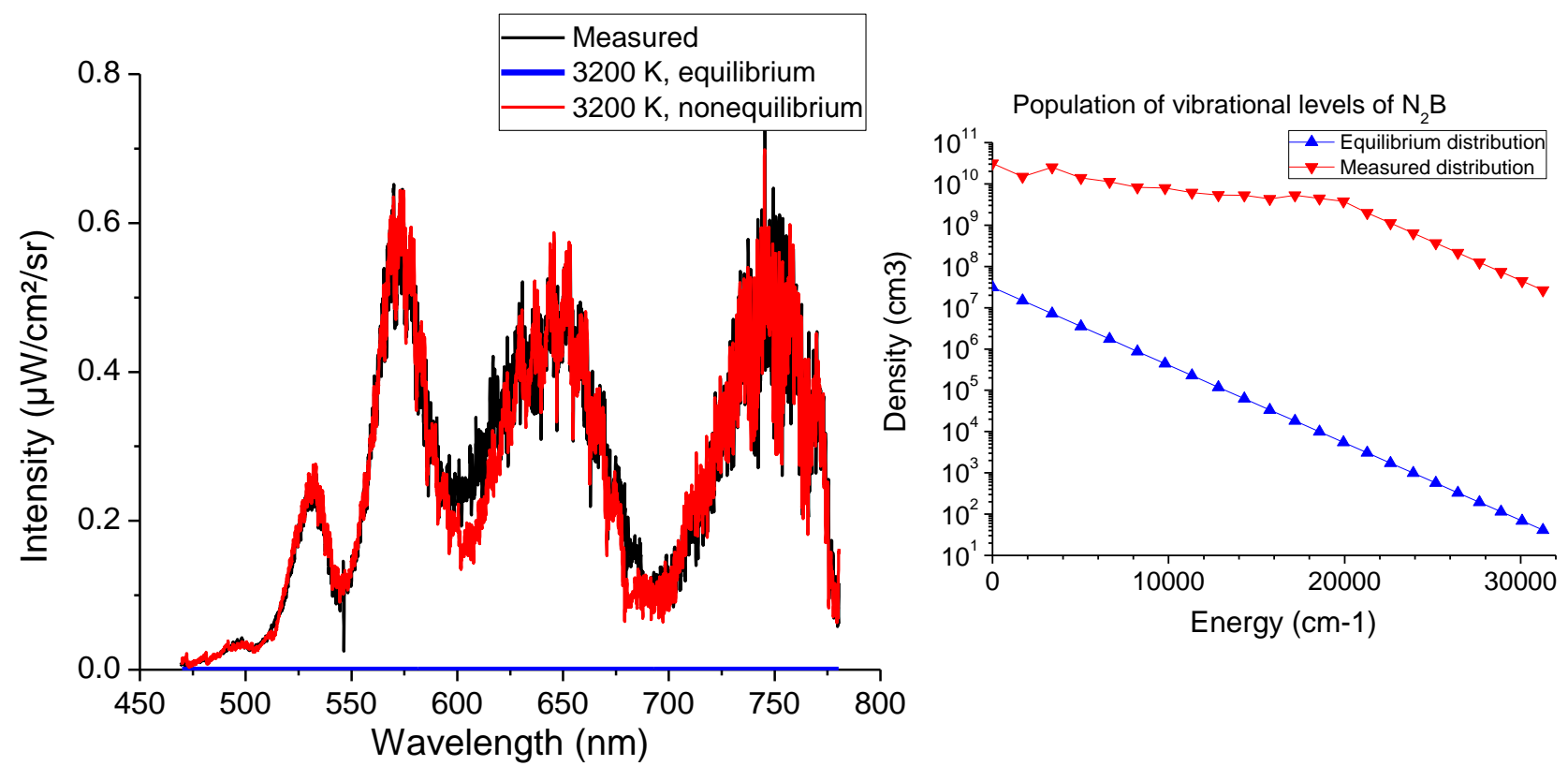

Figure 9: Left: Measured and theoretical spectra in the case of the $\mathrm{N}_{2} / \mathrm{Ar}$ at the exit of the $15 \mathrm{~cm}$ tube. The blue curve represents the computed SPECAIR spectrum assuming equilibrium and the red one is the computed spectrum assuming a non-equilibrium distribution of $\mathrm{N}_{2} \mathrm{~B}$. Right: The population of vibrational levels of $\mathrm{N}_{2} B$ taken in SPECAIR for calculation of theoretical spectra.

The good agreement between the spectrum assuming non-equilibrium and the measured one validates our measurement of the population distribution of the vibrational levels of $\mathrm{N}_{2} \mathrm{~B}$. Note that $\mathrm{N}_{2} \mathrm{~B}$ is highly overpopulated as compared to its equilibrium value because $\mathrm{N}$ does not have time to recombine in the tube due to the high velocity imposed. Note also that the vibrational population distribution in $\mathrm{N}_{2} \mathrm{~B}$ is no longer given by a Boltzmann distribution. It is therefore not possible to characterize the plasma using a single temperature. This is a challenge for CFD simulations as all vibrational levels should be included in the chemistry model to predict the correct radiative flux. This was also observed 
by Gessman et al. However, in their case, they calculated the overpopulation factors based upon the temperature measured using $\mathrm{N}_{2}{ }^{+}$emission. Given that this temperature is much higher than the Raman measurement, the overpopulation factors in their case were much lower. On the other hand, the populations measured by Gessman et al. are very close to those measured in the present work.

It is also possible to measure the atomic nitrogen density from this spectrum. As explained in Ref [7], the vibrational level $\mathrm{v}=13$ of $\mathrm{N}_{2}$ B is a predissociating state $N_{2}(B, v=13) \leftrightarrow N\left({ }^{4} S\right)+N\left({ }^{4} S\right)$. Considering that this reaction is equilibrated, the overpopulation of level $\rho_{\mathrm{N}_{2}, B, v=13}$ gives the overpopulation of atomic $\mathrm{N}$ via the equation: $\rho_{N}=$ $\sqrt{\rho_{\mathrm{N}_{2}, B, v=13}}$. With the Abel-inverted spectra, we measured the overpopulation of $\mathrm{N}_{2}, \mathrm{~B}, \mathrm{v}=13$ versus radius to infer the population of $\mathrm{N}$ across the tube diameter. Populations of $\mathrm{N}$ measured using this technique are plotted in figure 10.

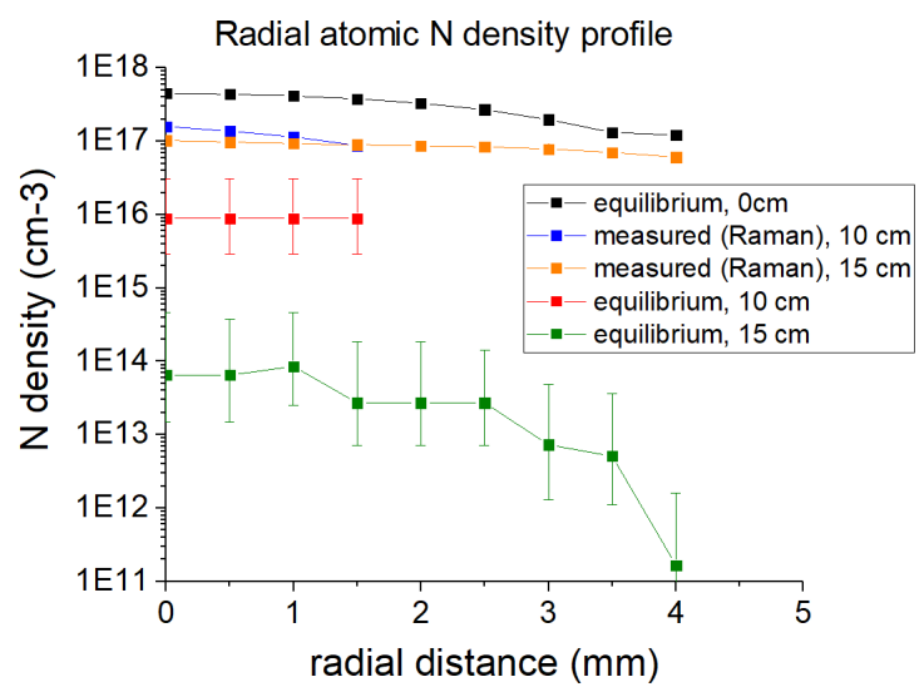

\section{Figure 10: radial population distribution of atomic nitrogen atoms, measured and calculated assuming an equilibrium population at the temperature measured using Raman spectroscopy}

The density of atomic nitrogen is highly above its equilibrium value at $10 \mathrm{~cm}$ and it does not change substantially between 10 and $15 \mathrm{~cm}$, suggesting that the $\mathrm{N}$ population is effectively frozen. Furthermore, a significant amount of enthalpy is stored in the unrecombined $\mathrm{N}$. This is an important consideration for the energy balance of the flow, as shown below. The nitrogen atomic density was also measured by Gessman et al in the center of the tube. They measured a density of $1.62 \pm 0.3 \cdot 10^{17} \mathrm{~cm}^{3}$ against $1.02 \pm 0.06 \cdot 10^{17} \mathrm{~cm}^{3}$ in our case. Both values are in good agreement despite using different temperatures conditions (we used temperature measured with Raman spectroscopy while Gessman et al used emission spectroscopy).

The enthalpy stored in unrecombined nitrogen atoms is given by the following relation:

$$
\Delta H_{\text {chem }}=\left(n_{N}-n_{N}^{*}\right) E_{\text {diss }_{N_{2}}}
$$

where $n_{N}$ is the measured number density of $\mathrm{N}, n_{N}^{*}$ is the equilibrium density of $\mathrm{N}$, and $E_{\text {diss }_{N_{2}}}$ the energy of dissociation of $\mathrm{N}_{2}$. This enthalpy is divided by the residence time of the gas in the tube to obtain a power:

$$
\Delta P_{\text {chem }}=\frac{\Delta H_{\text {chem }}}{\tau_{\text {residence }}}=\frac{\Delta H_{\text {chem }}}{\frac{x}{V_{\text {gas }}}}
$$

where $\mathrm{x}$ is the length of the tube $(10 \mathrm{or} 15 \mathrm{~cm})$ and $V_{\text {gas }}$ the mean velocity of the gas. Note that the velocity has not been measured directly and is estimated by matching the mass flow rate assuming a radial velocity profile self-similar to that of the measured temperature (justified by the fact that the Prandtl number, equal to 0.7, is close to unity). However, to account for any potential error due to this assumption, a large uncertainty (20\%) on the velocity is taken for each calculation involving this parameter. 
Based on the measured temperature profiles at the inlet and outlet of the tube, the power variation across the tube assuming equilibrium conditions is:

$$
\Delta P_{\text {gas (equilibrium) }}=\left[\int_{r=0 \mathrm{~mm}}^{r=5 \mathrm{~mm}} \rho_{\text {gas }} V_{\text {gas }} c_{p_{\text {gas }}} T_{\text {gas }} 2 \pi r d r\right]_{\text {outlet }}-\left[\int_{r=0 \mathrm{~mm}}^{r=5 \mathrm{~mm}} \rho_{\text {gas }} V_{\text {gas }} c_{p_{\text {gas }}} T_{\text {gas }} 2 \pi r d r\right]_{\text {inlet }}
$$

In addition, we also performed a calorimetric power balance on the water that traverses the water-cooled tube. This gives the power removed via the wall during the experiment and is equal to:

$$
\Delta P_{\text {water }}=\dot{m}_{\text {water }} * c_{p_{\text {water }}} *\left(T_{w o}-T_{w i}\right)
$$

where $\dot{m}_{w a t e r}$ is the measured water mass flow rate, $T_{w i}$ and $T_{w o}$ are the temperature of the water at the inlet and the outlet of the cooling tube, respectively.

Through a simple energy balance, this power must be equal to:

$$
\Delta P_{\text {water }}=\Delta P_{\text {gas }(\text { equilibrium })}-\Delta P_{\text {chemistry }}
$$

where $\Delta \mathrm{P}_{\text {chemistry }}$ represents the power stored due to chemical or thermal nonequilibrium: for example, the power stored in unrecombined nitrogen atoms.

Figure 11 shows the results obtained.
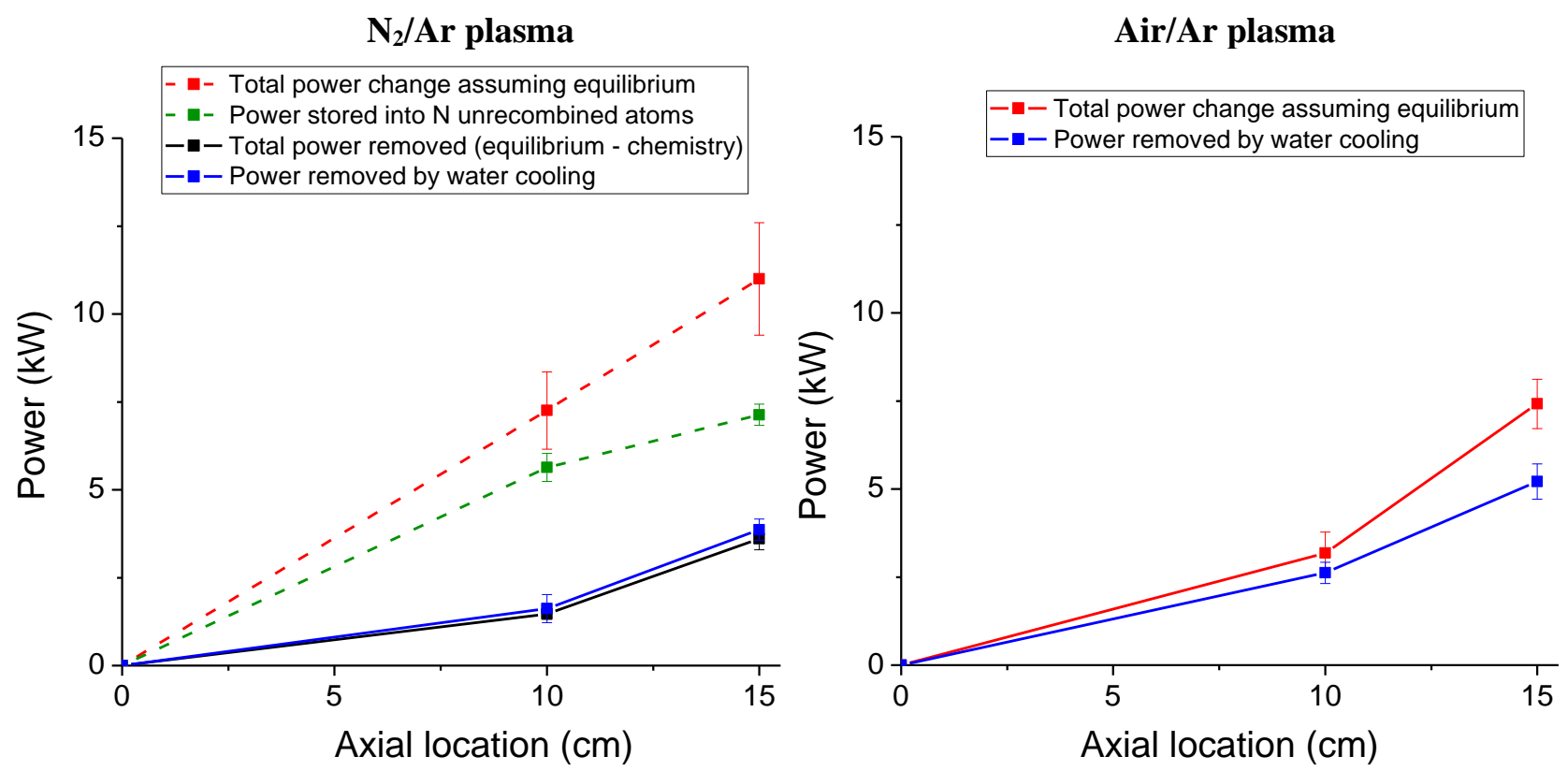

Figure 11: Power measurements using two different approaches for both plasmas cases.

Blue: measured power removed by water cooling.

Red: Power change between the exit and the inlet of the tube assuming equilibrium throughout the tube.

Green: power stored into unrecombined $\mathbf{N}$ atoms using the measured overpopulation factors.

Black: Red minus green curve: this represents the total power removed from the gas if only atomic nitrogen is responsible for the discrepancy in power. 
For the $\mathrm{N}_{2} / \mathrm{Ar}$ plasma the good agreement between the black and the blue curves indicates that the unrecombined $\mathrm{N}$ accounts for the missing energy. Note that all the calculations were done using the temperature measured via Raman spectroscopy. The chemical energy stored in the overpopulated vibrational levels of $\mathrm{N}_{2} \mathrm{~B}$ and $\mathrm{N}_{2} \mathrm{C}$, measured using emission spectroscopy, was also considered. However, this energy is negligible compared to the enthalpy stored in the unrecombined $\mathrm{N}$. This energy balance gives us confidence in the fact that the temperature of the gas is indeed the temperature measured with Raman. However, although these measurements are consistent with the Raman measurements, they do not explain the mechanism that causes the rapid cooling of the nitrogen/argon plasma.

In the case of an air/Ar plasma, we showed that the plasma stayed at equilibrium in the tube: thus, there is no energy stored into unrecombined nitrogen atoms. At $10 \mathrm{~cm}$, the power balance assuming equilibrium is in agreement with the power removed by the water as expected for an equilibrium plasma. However, there is a slight difference at $15 \mathrm{~cm}$. This can potentially be explained by unrecombined atoms near the wall. Near the wall, the rate of cooling is faster and is more likely to lead to a nonequilibrium distribution. However, due to the lower temperature close to the wall, it was not possible to measure possible overpopulations of atomic nitrogen using emission spectroscopy. Emission spectra were only measured for radii less than $3 \mathrm{~mm}$, where the emission was sufficiently strong.

These power balances show strong differences between the $\mathrm{N}_{2} / \mathrm{Ar}$ plasma and the air/Ar plasma. Both experiments are interesting for CFD codes as they have similar input conditions (same flow rates, temperatures, pressure...) but significantly different results at the tube outlet.

\section{Conclusions}

Experiments have been conducted in a plasma torch facility to study nonequilibrium recombining plasmas of nitrogen/argon and air/argon. These experiments may be used to validate CFD codes for atmospheric entry calculations.

Our results using emission spectroscopy are in agreement with earlier experimental results performed by Gessman et al [16]. We have also performed Raman scattering measurements to measure rotational and vibrational temperatures of $\mathrm{N}_{2}$ ground state. The vibrational and rotational temperatures obtained with Raman spectroscopy are in equilibrium and are much lower than those measured using emission spectroscopy. The higher temperature obtained by emission spectroscopy for the rotational distribution of levels of the $\mathrm{N}_{2}{ }^{+} \mathrm{B}$ state is likely due to the rapid quenching of excited ions states, which do not have time to relax to the background temperature of the gas. This has been observed by Studer and Vervisch [17]. Bruggeman et al [18] studied other scenarios where emission spectroscopy gives an incorrect measurement of the gas temperature. Thus, the Raman temperature measurements should be more reliable than those obtained with emission spectroscopy.

Given the temperature measured using Raman spectroscopy, we were able to calculate a simple energy balance which showed that a large portion of enthalpy remains in unrecombined atomic nitrogen.

An important conclusion of this work is that the experimental results remain in strong disagreement with the CFD simulations. This is likely to come from modeling issues on the radial heat transfer in CFD codes. Indeed, analysis on CFD results showed that not enough heat is transferred from the plasma close to the wall at $300 \mathrm{~K}$ and the plasma in the center of the tube.

The $\mathrm{N}_{2} /$ Ar plasma case is complex as it couples fluid mechanics and non-equilibrium chemistry. Our experiments can be used to uncouple these effects. Indeed, the air/Ar plasma case gives experimental results for a plasma that stays close to equilibrium conditions which is easier to model. Disagreement between experiments and CFD simulations for this case is likely to come from fluid mechanics modeling of the reacting flow. This should be further investigated to understand the disagreement between CFD simulations and experiments. Then simulations on the $\mathrm{N}_{2} / \mathrm{Ar}$ plasma case can be performed to validate the kinetic modeling. 
It is clear that this nonequilibrium experiment done using a simple configuration raises many interesting issues about nonequilibrium recombining nitrogen flows. These issues are currently the object of our continued investigations.

\section{Acknowledgments}

This work was supported by Ariane Group, under a CIFRE Ph.D. grant (number 42701092/20160218/JSE), with Dr. Laurent Visconti as Technical Monitor.

\section{References}

[1] P. A. Gnoffo, "Planetary-Entry Gas Dynamics," Annual Review of Fluid Mechanics, no. 31.1, pp. 459-494, 1999.

[2] C. O. Johnston, P. A. Gnoffo and A. Mazaheri, "Influence of Coupled Radiation and Ablation on the Aerothermodynamic Environment of Planetary Entry Vehicles," Radiation and Gas-Surface Interaction Phenomena in High Speed Re-Entry; 6-8- May 2013, 2013.

[3] S. Loehle, T. A. Hermann, F. Zander, H. Fulge and T. Marynomwski, "Ablation Radiation Coupling Investigation in Earth Re-entry Using Plasma Wind Tunnel Experiments," American Institute of Aeronautics and Astronautics, 2016/08/31 2014, 2014.

[4] M. E. MacDonald, C. M. Jacobs, C. O. Laux, F. Zander and R. G. Morgan, "Measurements of air plasma/ablator interactions in an inductively coupled plasma torch," J. Thermophys. Heat Transfer, vol. 29, pp. 12-23, 2015.

[5] S. D. McGuire, A. C. Tibère-Inglesse and C. O. Laux, "Infrared spectroscopic measurements of carbon monoxide within a high temperature ablative boundary layer," Journal of Physics D: Applied Physics, p. 49, 2016.

[6] C. O. Laux, R. J. Gessmann and C. H. Kruger, "lonizational Nonequilibrium Induced by Neutral Chemistry in Air Plasmas," AIAA journal, vol. 34, no. 8, pp. 1745-1747, Aug 1996.

[7] C. O. Laux, L. Pierrot and R. J. Gessman, "State-to-state modeling of a recombining nitrogen plasma experiment," Chemical Physics, vol. 398, pp. 46-55, 2012.

[8] G. V. Candler, C. O. Laux, R. J. Gessman and C. H. Kruger, "Numerical Simulation of a Nonequilibrium Nitrogen Plasma Experiment," 28th Plasmadynamics and Lasers Conference, Fluid Dynamics and Co-located Conferences, 1998.

[9] M. Nagulapally, D. Kolman, G. V. Candler, C. O. Laux, R. J. Gessman and C. H. Kruger, "Numerical Simulation of Nonequilibrium Nitrogen and Air Plasma Experiments," 29th AIAA, Plasmadynamics and Lasers Conference, Fluid Dynamics and Co-located Conferences, 1998.

[10] C. O. Johnston and A. M. Brandis, "Features of Afterbody Radiative Heating for Earth Entry," Journal of Spacecraft and Rockets, Vol. 52, vol. 52, pp. 105-119, 2015.

[11] C. O. Johnston, A. M. Brandis and K. Sutton, "Shock Layer Radiation Modeling and Uncertainty for Mars entry," 43rd AIAA Thermophysics Conference, Fluid Dynamics and Co-located Conferences, 2012.

[12] C. O. Laux, "Optical diagnostics and radiative emission of air plasmas," PhD Thesis, Stanford University, 1993.

[13] SPECAIR, Software Package, Ver. 3.0, SpectralFit S.A.S., www.spectralfit.com. See also [12]

[14] S. D. McGuire, A. C. Tibère-Inglesse and C. O. Laux, "Ultraviolet Raman spectroscopy of N2 in a recombining atmospheric pressure plasma," Plasma Sources Science and Technology, 2017. 
[15] C. O. Laux, R. J. Gessman, C. H. Kruger and S. P. Davis, "Rotational temperature measurements in air and nitrogen plasmas using the first negative system of N2+," Journal of Quantitative Spectroscopy and Radiative Transfer 68, vol. 68, pp. 473-482, 2001.

[16] R. J. Gessman, "An experimental investigation of the effects of chemical ionizational nonequilibrium in recombining atmospheric pressure air plasmas," PhD thesis.

[17] D. Studer and P. Vervisch, "Raman scattering,measurements within a flat plate boundary layer in an,inductively coupled plasma wind tunnel," Journal of Applied Physics, vol. 102, 2007.

[18] P. J. Bruggeman, N. Sadeghi, D. C. Schram and V. Linss, "Gas temperature determination from rotational lines in nonequilibrium plasmas: a review," Plasma Sources Science and Technology, vol. 23, 2014. 